\title{
Perspectiva da neurociência em comunicação
}

\author{
Everaldo Pereira \\ Universidade Metodista de São Paulo, Brasil \\ E-mail: everaldo.creative@gmail.com
}

\begin{abstract}
Resumo
Pesquisa bibliográfica com objetivo de consolidar o estágio atual da intersecção entre neurociência e comunicação. Usamos um referencial teórico multidisciplinar com o objetivo de oferecer insights investigativos e chaves de entendimento. Dividido em três partes: compreender a neurociência e sua interface com a comunicação; investigar as tecnologias

em neurociência e suas aplicações em comunicação; e avaliar a viabilidade do uso da metodologia de Design Science Research na interface comunicaçãoneurociência. Compreendemos que há espaço para uma investigação sistemática e de que é viável o uso dessa metodologia, considerando uma orientação pragmática utópica.
\end{abstract}

Palavras-chave: neurociência em comunicação; neurocomunicação; design science research; pragmatismo utópico.

\section{Perspective of neuroscience in communication}

\begin{abstract}
Bibliographical research aiming to consolidate the current stage of the intersection between neuroscience and communication. We use a multidisciplinary theoretical framework with the objective of offering investigative insights and keys of understanding. Divided into three parts: understanding neuroscience and its interface with communication; to

plications in communication; and evaluate the feasibility of using the Design Science Research methodology in the communication-neuroscience interface. We understand that there is room for a systematic investigation and that the use of this methodology is feasible, considering a utopian pragmatic orientation.
\end{abstract} investigate neuroscience technologies and their ap-

Keywords: neuroscience in communication; neurocommunication; design science research; utopian pragmatism.

Data de submissão: 2018-01-24. Data de aprovação: 2018-04-26.

A Revista Estudos em Comunicação é financiada por Fundos FEDER através do Programa Operacional Factores de Competitividade - COMPETE e por Fundos Nacionais através da FCT - Fundação para a Ciência e a Tecnologia no âmbito do projeto Comunicação, Filosofia e Humanidades (LabCom.IFP) UID/CCI/00661/2013.
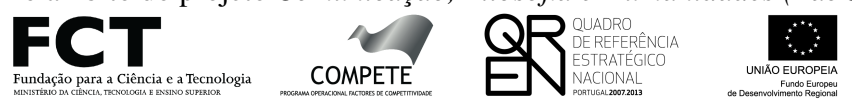

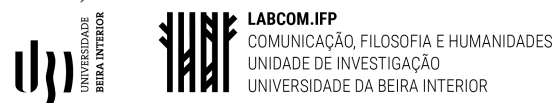




\section{INTRODUÇÃO}

Detendemos realizar uma pesquisa bibliográfica para compreender o "estado da arte" da perspectiva neurocientífica em comunicação, com o objetivo de buscar caminhos investigativos e chaves de entendimento para os fenômenos sociais desse campo de estudo.

Compreendemos que ainda é insipiente o número de pesquisas e abordagens a partir dessa perspectiva, principalmente pesquisas em que a comunicação social é "agente" na investigação e não apenas "cliente" dos resultados obtidos em neurociência.

Pretendemos dividir o trabalho em três partes: compreender a neurociência e sua interface com a comunicação; investigar as tecnologias em neurociência e suas aplicações em comunicação; e avaliar a viabilidade do uso da metodologia de Design Science Research na interface comunicaçãoneurociência.

\section{NEUROCIÊNCIA E SUA INTERFACE COM A COMUNICAÇÃO}

Os interesses investigativos dos diversos campos do conhecimento, inevitavelmente, são frutos do zeitgeist e, nesse sentido, no campo da comunicação, as investigações passaram por algumas mudanças naturais ao longo das décadas, com evoluções do pensamento comunicacional culminando numa pluralidade de percepções.

Atualmente o desenvolvimento tecnológico possibilitado pelos avanços tecnocientíficos tem se consolidado no que Harari (2015) denominou de Revolução Científica, em comparação com a Revolução Cognitiva de cerca de 70.000 anos e a Revolução Agrícola de cerca de 10.000 anos. Neste zeitgeist tecnocientífico, a comunicação pode interagir com muitos campos do conhecimento para abarcar novas visões e desenvolver novos referenciais teóricos e novas metodologias para além dos referenciais e metodologias consolidadas.

Vários autores, entre eles Kaku (2011), Kurzweil (2013), Zarkadakis (2015), Nicolelis (2011), entre outros, têm demonstrado o avanço da neurociência neste início de século, a partir, principalmente de tecnologias não-invasivas, como demonstraremos mais à frente neste trabalho. Por outro lado, autores como Squirra (2012), Lemos (2005), Castro; Tome; Barbosa Filho (2005), entre outros, analisam como as tecnologias estão imbricadas na comunicação. Mais recentemente, Squirra (2016) debate como a tecnologia e a evolução poderão levar o campo da comunicação para a noosfera, ou seja, a esfera do pensamento humano. Compreender a interface entre neurociência e comunicação, portanto, torna-se um escopo investigativo relevante para ampliar nosso campo de conhecimento.

Do nosso ponto de vista, o que faz da neurociência um foco de investigação atual para as pesquisas em comunicação são as tecnologias não-invasivas de imagens do sistema nervoso. A popularização dessas tecnologias na medicina, como veremos adiante, não datam de mais de 20 anos. Assim, anteriormente, as contribuições da neurociência em comunicação dependiam de investimentos altíssimos e disponibilidade de equipamentos. Além disso, a própria neurociência avançava devagar porque não se conheciam todos os meandros do sistema nervoso, a depender apenas de tecnologias invasivas ou de cérebros mortos. 
Historicamente o interesse pelo cérebro é tão antigo quanto o ato de pensar. Segundo Bear, Connors e Paradiso (2002), há evidências de trepanações ${ }^{1}$ em crânios de 7.000 anos. No entanto, é entre os séculos XIX e XX que a doutrina neuronal ganha força com as descobertas separadamente de Golgi e Cajal (Gazzaniga; Ivry; Mangun, 2006) sobre a coloração de neurônios e os mecanismos da cada neurônio, respectivamente.

No desenrolar do século XX, com o avanço de diversas técnicas e campos de conhecimento, como a psicologia, novas abordagens foram se incorporando às ciências do sistema nervoso. De início surgiu a ideia do localizacionismo, que entende os comportamentos como oriundos de um lugar específico no cérebro. Logo em seguida surgiu a escola holística, influenciada pelas bases da Gestalt, de que o todo é mais do que a soma das partes. Ainda hoje há quem encontre contradições nas duas linhas de pensamento, mas nota-se um início de consenso, segundo Gazzaniga, Ivry; Mangun (2006), Bear, Connors, Paradiso (2002), Nicolelis (2011), entre outros, de que as duas linhas tem suas evidências positivas e alguns pontos incorretos e que, portanto, são complementares e não excludentes.

Hoje os conhecimentos consolidados em neurociência nos permitem investigações em diferentes níveis de análise que, segundo Bear, Connors, Paradiso (2002), são: moleculares, celulares, de sistemas, comportamentais e cognitivos. O nível molecular investiga as diversas moléculas presentes no sistema nervoso, algumas exclusivas do sistema nervoso. O nível celular investiga os tipos de neurônios e seu funcionamento individual. O nível de sistemas investiga como constelações de neurônios, ou populações de neurônios como denomina Nicolelis (2011), realizam determinada função comum. Os níveis comportamental e cognitivo, parece-nos, são os que mais podem contribuir para uma interface neurociência-comunicação, neste momento, porque o comportamental investiga como sistemas neurais trabalham juntos para produzir comportamentos integrados, e o cognitivo, que deseja responder como o cérebro cria a mente, ou seja, a consciência.

A temática consciência - e a dualidade mente-cérebro que a acompanha - gera controvérsias a centenas de anos. "Nunca na ciência tantos se dedicaram tanto para criar tão pouco consenso", nas palavras de Kaku (2015). Este mesmo autor propõe o que chama de "teoria espaço-tempo da consciência":

Consciência é o processo de criar um modelo do mundo usando múltiplos ciclos de feedback em vários parâmetros (por exemplo, temperatura, espaço, tempo e em relação a outros), a fim de atingir uma meta (por exemplo, encontrar parceiros, comida, abrigo). (Kaku, 2015)

Nesse sentido, diz o autor, difere do modelo de mundo dos animais baseado no espaço e na relação com outros animais, enquanto os humanos criam um modelo de mundo baseado no espaço, na relação com outros e no tempo [grifo nosso]. Kaku desenvolve esse conceito a partir de uma visão fisicista sobre a Teoria do Cérebro Trinuo, de Paul MacLean, ([1967]1990), que entende o cérebro como um produto do processo evolutivo desde o réptil, passando pelo mamífero até o humano.

1. Furo cirúrgico no crânio de uma pessoa viva com objetivo de cura (Bear; Connors; Paradiso, 2002). 


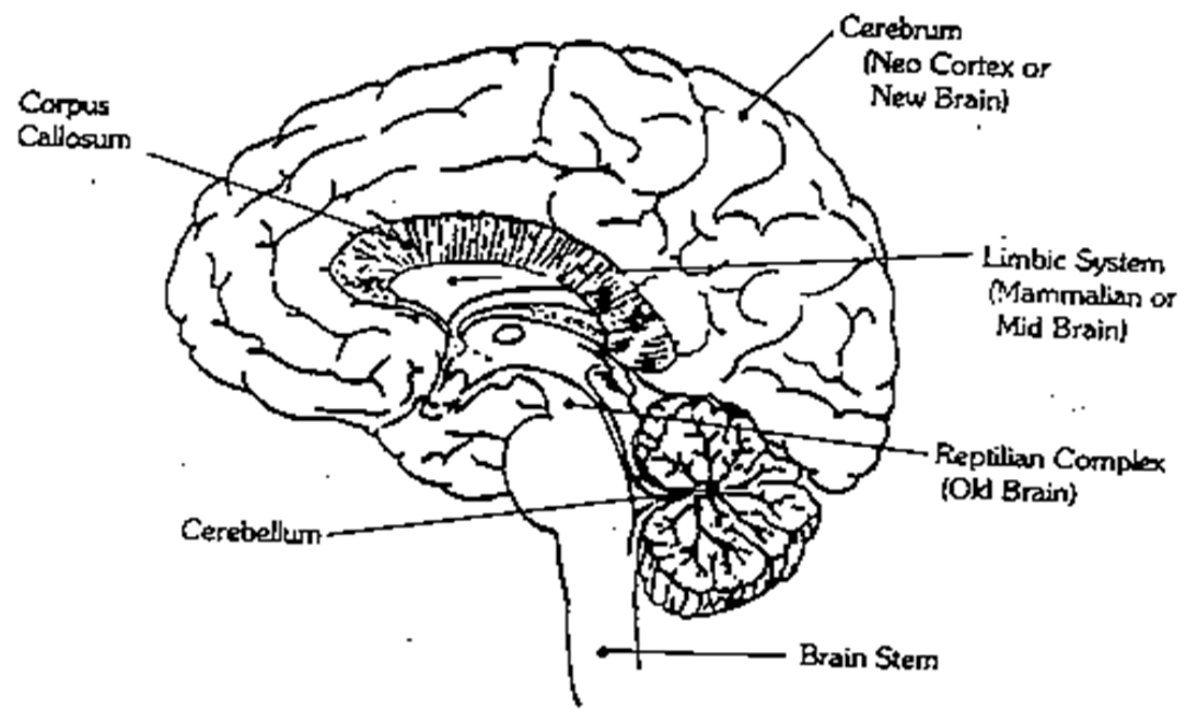

Figura 1. Teoria do cérebro triuno

Fonte: Maclean, 1990

Para evidenciar suas proposições, Kaku elenca níveis de consciência por diferentes espécies que correspondem às estruturas cerebrais denominadas tronco cerebral (réptil), sistema límbico (mamífero) e córtex pré-frontal (humano). Nesse sentido a consciência humana é aquela capaz de criar um sentido de mundo a partir do espaço, das relações sociais e do tempo. A comunicação, se seguirmos nessa linha, evoluiu para abarcar essas relações sociais de tempo-espaço.

Tabela 1. Níveis de consciência de diferentes espécies

\begin{tabular}{llll} 
Nível & Espécie & Parâmetro & Estrutura Cerebral \\
\hline 0 & Plantas & Temperatura, luz solar & Nenhuma \\
I & Répteis & Espaço & Tronco cerebral \\
II & Mamíferos & Relações sociais & Sistema límbico \\
III & Humanos & Tempo (especialmente futuro) & Córtex pré-frontal \\
\hline
\end{tabular}

Fonte: Kaku, 2015 p. 49

Do nosso ponto de vista, isso tem fundamental importância para os estudos de comunicação porque é também a partir das percepções de tempo, de espaço e de relações sociais que se desenrola o interpretar simbólico. Consciência e comunicação estão imbricados desde tempos remotos quando o cérebro dos humanos mais primitivos, ao longo de milhares de anos e por várias razões, começou a aumentar em relação aos outros mamíferos. Segundo Harari (2015) paralelamente, o corpo foi ficando mais ereto e os quadris, mais estreitos. Consequentemente as grávidas precisavam ter filhos mais cedo, estes ainda com sistemas subdesenvolvidos em comparação a outros animais. Filhos subdesenvolvidos demandam mais atenção das mães que não tem como prover a 
subsistência dos dois sem ajuda. Assim, as relações sociais, típicas dos mamíferos, se tornaram mais sólidas nos humanos e a comunicação, possibilitada por cérebros mais cognitivos, foi a base para o surgimento de grandes aglomerados humanos. A partir daí, as tecnologias comunicacionais permitiram ampliar as relações sociais por meio da colaboração humana no espaço e no tempo: tribos, cidades, nações e a ideia da aldeia global, com passado, presente e futuro. Comunicar é conviver, nas palavras de Wolton (2011).

Nos diversos níveis de análise cerebral, neurocientistas e psicólogos tem se debruçado nas últimas décadas para traçar um perfil do funcionamento do cérebro. Mas, como avaliado por diversos filósofos da ciência, os fenômenos podem ser explicados por diversas formas. A motivação humana, característica de diversas interações comunicacionais, pode ser observada por diferentes aspectos neuronais, no entanto, muitos ainda carentes de consolidação científica. Um exemplo é gostar de assistir a um vídeo e querer assistir a um vídeo. Bear, Connors e Paradiso (2002) notam que gostar e querer podem fazer parte de um único processo mental, mas são mediados por diferentes circuitos no encéfalo. Assim, a bibliografia em neurociência aborda aspectos físicos e biológicos do funcionamento cerebral para explicar diferentes aspectos de comportamentos humanos.

O que é possível inferir pelos levantamentos bibliográficos em neurociência é de que o campo tende a se expandir nos próximos anos, abrindo possibilidades investigativas interdisciplinares. Para a comunicação, um olhar sob a perspectiva neurocientífica é salutar na medida em que as tecnologias digitais interconectadas e conectadas ao cérebro por meio de tecnologias não-invasivas ou por meio de implantes de microcomponentes podem levar a comunicação humana para outra dimensão, como exposto por Squirra (2016).

Tabela 2. Recentes investigações em neurociência e suas interfaces com a comunicação

\begin{tabular}{|c|c|c|c|}
\hline Investigação & $\begin{array}{l}\text { Intersecção } \\
\text { comunicativa }\end{array}$ & Perspectivas comunicacionais & Referências \\
\hline $\begin{array}{l}\text { Ler a mente a partir de ondas cere- } \\
\text { brais para formar padrões de rostos } \\
\text { humanos }\end{array}$ & $\begin{array}{l}\text { Percepção visual, } \\
\text { imaginário }\end{array}$ & $\begin{array}{l}\text { brain-net, inteligência artificial, In- } \\
\text { terfaces cérebro-computador, desin- } \\
\text { termediação }\end{array}$ & $\begin{array}{l}\text { Lee; Kuhl, } \\
2016\end{array}$ \\
\hline $\begin{array}{l}\text { Fala é resultado da interação dos } \\
\text { dois hemisférios cerebrais }\end{array}$ & Linguagem & Ciberpatia, desintermediação & $\begin{array}{l}\text { Cogan et al., } \\
2014\end{array}$ \\
\hline $\begin{array}{l}\text { Comunicação cérebro a cérebro } \\
\text { usando tecnologias não invasivas }\end{array}$ & $\begin{array}{l}\text { Teoria da } \\
\text { informação }\end{array}$ & $\begin{array}{l}\text { Cibercomunicação, brain-net, ciber- } \\
\text { patia, desintermediação }\end{array}$ & $\begin{array}{l}\text { Grau et al., } \\
2014\end{array}$ \\
\hline $\begin{array}{l}\text { Mapa semântico da fala no córtex } \\
\text { cerebral }\end{array}$ & $\begin{array}{l}\text { Semiótica, } \\
\text { imaginário }\end{array}$ & $\begin{array}{l}\text { Brain-net, Interfaces cérebro-com- } \\
\text { putador }\end{array}$ & $\begin{array}{l}\text { Huth et al., } \\
2016\end{array}$ \\
\hline $\begin{array}{l}\text { Reconstrução de experiências visu- } \\
\text { ais da atividade cerebral evocadas } \\
\text { por filmes naturais }\end{array}$ & $\begin{array}{l}\text { Percepção visual, } \\
\text { linguagem visual, } \\
\text { imaginário }\end{array}$ & $\begin{array}{l}\text { Cibercomunicação, entretenimento, } \\
\text { inteligência artificial, Interfaces cé- } \\
\text { rebro-computador, ciberpatia }\end{array}$ & $\begin{array}{l}\text { Nishimoto } \\
\text { et al., } 2011\end{array}$ \\
\hline $\begin{array}{l}\text { Aumento de consumo a partir da } \\
\text { aplicação de laser no cérebro }\end{array}$ & $\begin{array}{l}\text { Propaganda, } \\
\text { consumo }\end{array}$ & Cibercomunicação & $\begin{array}{l}\text { Reardon, } \\
2016\end{array}$ \\
\hline
\end{tabular}

Fonte: elaborada pelo autor 
Pesquisas recentes, como demonstradas na Tabela 2, indicam que os avanços em neurociência e áreas correlatas podem mudar os processos comunicativos como os conhecemos. Cada vez mais pesquisas demonstram como é possível compreender funções cerebrais distintas, localizando padrões cerebrais que nos permitem "ler" e "reproduzir" pensamentos por meio de máquinas, ou ainda, de cérebro a cérebro. Neste escopo, a interface cérebro-computador, conexões sem fio e conteúdos em nuvem, permitem supor uma comunicação cada vez mais desintermediada.

A desintermediação pode ser entendida como um processo comunicacional de acesso e emissão de conteúdos sem a participação da indústria midiática como a conhecemos. Além da desintermediação, é possível supor que o avanço neurocientífico poderá proporcionar uma conexão de cérebros, uma brain-net (Nicolelis, 2011; Kaku, 2015), uma rede de cérebros conectados. É possível que no futuro, esses conglomerados evoluam (no sentido darwinista de adaptação) para gestores de "neuroconteúdos" em nuvem, assim como o Google tem se tornado uma gigante midiática que não produz quase nenhum conteúdo. Se gerir o conhecimento de nós a partir de nossos dados e informações postados em rede e vender aos anunciantes, pôde levar essa empresa ao topo, o que dizer de gerir nossos próprios pensamentos e sugerir "rankings de pensamentos" direto em nossos cérebros?

Os avanços tecnocientíficos em inteligência artificial e em neurociência poderão permitir o desenvolvimento da consciência artificial, ou neural machines (Zarkadakis, 2015) o que mudará aspectos profundos da sociabilização. Mudará a comunicação entre humanos; a comunicação entre humanos e outros animais; a comunicação entre humanos e máquinas; e a comunicação entre máquinas. Haverá sociedades de máquinas conscientes que criarão novas dimensões comunicacionais?

Leitura de padrões neurais e reprodução em outros cérebros por meio de conexões sem fio tem se tornado um tema importante de pesquisas em várias universidades ao redor do mundo. Isso tem sido comparado, segundo Kaku (2014) à telepatia, palavra cercada de significados e comumentemente associada ao charlatanismo. Do grego tele, distância, e pathia, sentimento. Não deixa de ser verdade que hoje é possível captar rudimentos de pensamentos e transmiti-los à distância para outro cérebro em um outro lugar do mundo por meio da internet, mas, parece-nos que a neurociência e a física tem possibilitado uma ciberpatia, uma vez que o prefixo "ciber" com sua aura tecnológica coringa, adapta-se melhor à explicação das evidências observadas, como na pesquisa Conscious brain-to-brain communication in humans using non-invasive Technologies ${ }^{2}$, de Grau et al. (2014), que demonstra como a percepção luminosa pode ser enviada e compreendida de cérebro para cérebro à distância.

2. Comunicação consciente cérebro a cérebro em humanos usando tecnologias não invasivas [tradução nossa]. 


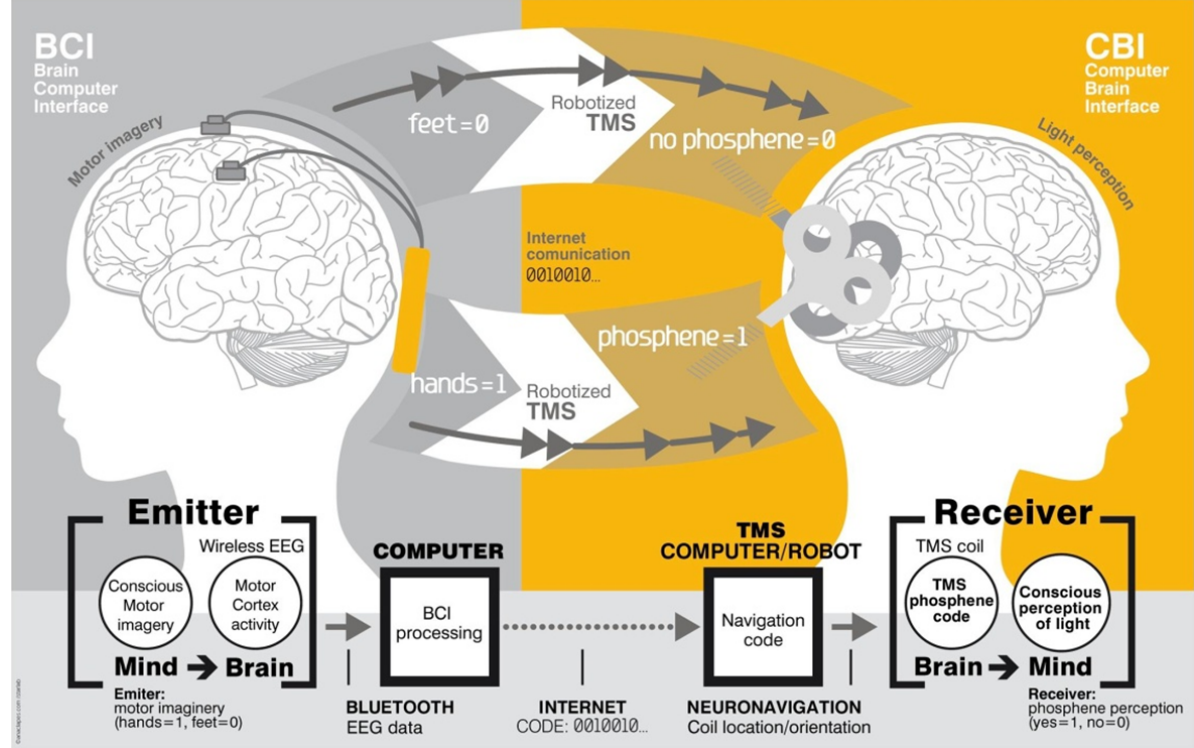

Figura 2. Conscious Brain-to-Brain Communication in Humans Using Non-Invasive Technologies Fonte: Grau et al., 2014

As ciências sociais, de modo geral, e particularmente a comunicação, historicamente tem demonstrado pouca investigação neste campo interdisciplinar. Kawano (2014) em trabalho apresentado no XXXVII Congresso Brasileiro de Ciências da Comunicação, em Foz do Iguaçu, demonstrou por meio de pesquisa bibliométrica que apenas 3,6\% dos documentos pesquisados nas bases científicas Scopus e Web of Science apresentavam a relação entre neurociências e ciências sociais.

Algumas iniciativas nesse campo podem ser observadas, como o Media Neuroscience Lab, um laboratório de pesquisa em neurociência e comunicação, tendo como principal pesquisador o prof. René Weber, da Universidade da Califórnia Santa Bárbara, que explica:

smallSimplesmente, o modelo que defendemos argumenta que qualquer teoria completa do comportamento humano no contexto da comunicação de massas especifica relações empiricamente observadas entre as variáveis, os substratos neurais dessas variáveis e uma explicação sonora (preferencialmente evolutiva) para esses substratos neurais. A maioria das teorias atualmente populares na pesquisa de comunicação de massa fornece relatos descritivos das relações observadas entre variáveis, às vezes com apelos ligeiramente explicados à ciência cognitiva ou modelos da mente (em oposição aos modelos de função cerebral).[tradução nossa] (Weber; Mathiak; Sherry, 2009 , p. 44-45) ${ }^{3}$

3. Simply, the model we advocate argues that any complete theory of human behavior in the mass communication context specify empirically observed relationships among variables, the neural substrates of those variables, and a sound (preferably evolutionary) explanation for those neural substrates. Most theories that are currently popular in mass communication research provide descriptive accounts of observed relationships among variables, sometimes with marginally explicated appeals to cognitive science or models of the mind (as opposed to models of brain function). 
Nota-se que as chaves de entendimento são de corrente teórica positivista, de viés biológico. Weber defende um modelo que se concentra no uso dos meios primeiramente como uma função biológica, e não social. "A função biológica nos fornece a entrada em mecanismos explicativos ao contrário da descrição explicativa", expõe Weber (p. 45). Entendemos que uma intersecção de corrente teórica sociológica alinhada a esses entendimentos de viés biológico pode contribuir para o entendimento de variáveis complexas de comunicação. As questões metodológicas sedimentadas da comunicação, como questionários, entrevistas, grupos focais, observações participantes, pesquisa documental, etnografia, internetgrafia, análise de discurso, análise de conteúdo, entre outras, podem ser ampliadas com percursos metodológicos enriquecidos pela neurociência e pela psciologia cognitiva.

\section{TECNOLOGIAS de PESQUiSA EM NEUROCIÊNCIA E SUAS RELAÇões COM A COMUNICA-} ÇÃO

Como dissemos, o que possibilitou o avanço da pesquisa neurocientífica, sem dúvida, são as inovações tecnológicas de imagens que tem por base a física. São usadas para várias finalidades, desde diagnósticos médicos a detectores de mentira. Para o presente trabalho focamos na investigação desses aparelhos para decodificação cerebral.

Tabela 3. Principais tecnologias para decodificação cerebral

\begin{tabular}{|c|c|c|c|}
\hline Tecnologias & Função & $\begin{array}{l}\text { Possibilidades investigativas } \\
\text { em comunicação }\end{array}$ & Pontos a serem observados \\
\hline $\begin{array}{l}\text { Eletroencefalograma } \\
\text { (EEG) }\end{array}$ & $\begin{array}{l}\text { Medir a capacidade ge- } \\
\text { ral do cérebro em dife- } \\
\text { rentes estados de consci- } \\
\text { ência por sinais elétricos. }\end{array}$ & $\begin{array}{l}\text { Controle de interfaces digi- } \\
\text { tais por pensamento; Inves- } \\
\text { tigar a comunicação em es- } \\
\text { tados de inconsciência. }\end{array}$ & $\begin{array}{l}\text { Deficiência em resolução } \\
\text { espacial, Não indicado para } \\
\text { investigações de maior } \\
\text { complexidade. }\end{array}$ \\
\hline $\begin{array}{l}\text { Imagem por resso- } \\
\text { nância magnética } \\
\text { (IRM) }\end{array}$ & $\begin{array}{l}\text { Mostrar como as várias } \\
\text { regiões do cérebro intera- } \\
\text { gem umas com as outras } \\
\text { por meio de imagem de- } \\
\text { talhada em 3D. }\end{array}$ & $\begin{array}{l}\text { Processos cognitivos e com- } \\
\text { portamentais da co- mu- ni- } \\
\text { cação. Percepção vi- sual. } \\
\text { Análise de design. }\end{array}$ & $\begin{array}{l}\text { Altos custos e tem menor } \\
\text { capacidade de resolução } \\
\text { temporal }\end{array}$ \\
\hline $\begin{array}{l}\text { Tomografia por emis- } \\
\text { são de pósitrons } \\
\text { (TEP) }\end{array}$ & $\begin{array}{l}\text { Calcula o fluxo de ener- } \\
\text { gia no cérebro. }\end{array}$ & $\begin{array}{l}\text { Avaliação de ações neuro- } \\
\text { nais de alta complexidade da } \\
\text { comunicação. Base para in- } \\
\text { teligência artificial. }\end{array}$ & $\begin{array}{l}\text { Altos custos e levemente ra- } \\
\text { dioativa. Não se deve sub- } \\
\text { meter uma pessoa ao pro- } \\
\text { cedimento mais de uma vez } \\
\text { por ano. }\end{array}$ \\
\hline Magnetismo & $\begin{array}{l}\text { Desligar com segurança } \\
\text { partes específicas do cé- } \\
\text { rebro sem necessidade de } \\
\text { técnicas invasivas. }\end{array}$ & $\begin{array}{l}\text { Localizar regiões que intera- } \\
\text { gem no processo comunica- } \\
\text { tivo e avaliar os efeitos co- } \\
\text { municativos ao desligá-las. }\end{array}$ & $\begin{array}{l}\text { Dificuldades para investigar } \\
\text { partes profundas do cére- } \\
\text { bro, como o sistema lím- } \\
\text { bico. }\end{array}$ \\
\hline
\end{tabular}

Fonte: elaborada pelo autor. 
Podemos observar pela Tabela 3 que o eletroencefalograma (EEG), como uma tecnologia capaz de medir a capacidade neuronal, rastrear grandes áreas cerebrais, permite várias intersecções com a comunicação, como controlar displays com o pensamento, a partir de neuroheadsets ${ }^{4}$, como o e-motiv, um equipamento leve e prático desenvolvido pela pesquisadora Tan Lee para monitoramento cerebral e avaliação cognitiva com custos baixos. A imagem por ressonância magnética (IRM) possibilita a investigação em tempo real de ações neurais de processos cognitivos, como percepção. No conhecido estudo Neural correlates of behavioral preference for culturally familiar drinks ${ }^{5}$, McClure et al. (2004), demonstra as evidencias neurológicas da percepção da imagem de marca. Os pesquisadores realizaram testes-cegos e testes com rótulos das marcas Coca-Cola e Pepsi e avaliaram alterações na região cerebral indicativa de felicidade. Os pesquisadores comprovaram que a percepção de imagem de marca altera estados mentais, independentemente das características físicas do produto. As tecnologias de fIRM, ou imagem por ressonância magnética funcional, uma variação da IRM, permitirão um "dicionário de pensamentos" (Kaku, 2011), de modo que cada objeto do mundo físico tenha uma correspondência de um-para-um para uma imagem de fMRI, diz o autor. Assim, pode-se decifrar o objeto que a pessoa está pensandoa partir de um computadores lendo milhares de padrões fMRI em tempo real. Poderemos decodificar a consciência de uma pessoa. É o que nos mostra o trabalho de Crew (2016):

A máquina conseguiu reconstruir cada face [rosto] com base na atividade de duas regiões distintas no cérebro: o giro angular (ANG), envolvido em vários processos relacionados à linguagem, processamento de números, consciência espacial e formação de memórias vívidas; E o córtex occipitotemporal (OTC), que processa pistas visuais. ${ }^{6}$

A tomografia por emissão de pósitrons (TEP), por sua vez, parece-nos a mais difícil de ser aplicada em pesquisas em comunicação neste momento, devido ao alto custo e à exposição do participante à radiação leve. No entanto esta tecnologia possibilita uma quantificação de informação e a capacidade de estudar uma gama muito maior de funções cerebrais, que incluem principalmente a neurotransmissão e os neurorreceptores. (Costa; Oliveira; Bressan, 2001). É possível que num futuro próximo, com o avanço das tecnologias de detecção por imagem nuclear, os custos e riscos envolvidos diminuam e as aplicações em neurocomunicação sejam mais comuns, auxiliando, por exemplo, nas bases para o desenvolvimento de inteligência artificial.

\section{Viabilidade do uso de Design Science Research PARA A INTERSECÇão ENTRE NEURo- CIÊNCIA E COMUNICAÇÃ̃o}

Compreendendo, portanto, o potencial da intersecção entre neurociência e comunicação, fazse juz avaliar novas possibilidades metodológicas para a descrição e interpretação de objetos de

4. Aparelhos portáteis e não-invasivos de decodificação cerebral por EEG.

5. Correlatos neurais de preferência comportamental para bebidas familiares preferidas culturalmente [tradução nossa]

6. The machine managed to reconstruct each face based on activity from two separate regions in the brain: the angular gyrus (ANG), which is involved in a number of processes related to language, number processing, spatial awareness, and the formation of vivid memories; and the occipitotemporal cortex (OTC), which processes visual cues [tradução nossa]. 
estudos nesta visão. Ao nos depararmos com a já ampla gama de opções metodológicas, como bibliográfica, entrevistas, biográficas, etnográficas, pesquisa-ação, internetgrafia, de opinião, grupos focais, semióticas, estudos de caso, auditorias de imagem, documental, análise de conteúdo, análise de discurso, hermenêutica, análise de discurso, entre outras (Duarte; Barros, 2006), compreendemos que novas metodologias são necessárias para produzir e interpretar os diferentes tipos de variáveis envolvidas.

Para tanto avaliamos a viabilidade do uso de Design Science Research como uma metodologia para investigação que também possa contribuir com essa nova perspectiva. Design Science Research é "o método que fundamenta e operacionaliza a condução da pesquisa quando o objetivo a ser alcançado é um artefato ou prescrição" (Dresch, 2015, p. 67). Por artefatos, entenda-se "como algo que é construído pelo homem, ou objetos artificiais que podem ser caracterizados em termos de objetivos, funções e adaptações" (Simon apud Dresch, 2015).

Nesse sentido, a Design Science Research é um método alicerçado no pragmatismo utópico, orientado à solução de problemas, a fim de compreendê-los e construir e avaliar artefatos que permitam melhorar determinadas situações. Em comparação com as ciências mais tradicionais, como as naturais e as sociais que buscam explicar, descrever, explorar ou predizer fenômenos e suas relações, a Design Science é orientada à prescrição, com foco na solução, tendo como resultado uma regra tecnológica testada e aprovada. O propósito da design Science, portanto é produzir sistemas que ainda não existem ou melhorar sistemas existentes. Segundo Dresch, (2015), os sete critérios fundamentais dessa metodologia são: compreender design como artefato; foco em problemas relevantes; executar a avaliação do design; deve prover contribuições claras e verificáveis; deve ser baseada em métodos rigorosos; compreender design como um processo de pesquisa; e comunicar a pesquisa.

Partindo então das perspectivas comunicacionais e neurociência propostas na tabela 2, poderemos avaliar as seguintes possibilidades investigativas em Design Science Research na comunicação:

- brain-net - construção e validação de dicionários digitais de pensamentos; de meios de acesso à bancos de pensamentos; de organizadores de pensamentos; e novas organizações midiáticas baseadas em pensamentos.

— inteligência artificial - construção e validação de sistemas de conversação artificial; de gestores de conteúdo por algoritmos; de apresentadores artificiais de entretenimento; de jornalistas à base de IA; de romancistas à base de IA; de filmes editados por consciência coletiva;

- Interfaces cérebro-computador - de equipamentos de comunicação para transhumanistas; de novas interfaces de organização de conteúdo digital;

- Desintermediação - construção e validação de substitutos aos displays tradicionais; de novos modelos de gestores de conteúdo em nuvem em substituição aos modelos tradicionais de organizações de mídia;

- Ciberpatia - construção e validação de novas redes de comunicação ciberpáticas; de modelos reguladores do tráfego de informações ciberpáticas;

Em relação às possibilidades investigativas em comunicação propostas na Tabela 3, poderemos avaliar as seguintes prescrições a partir da Design Science Research na comunicação: 
- Prescrições para o controle de interfaces digitais por pensamento; Prescrições na investigação da comunicação em estados de inconsciência.

- Prescrições de análise crítica dos processos cognitivos e comportamentais da comunicação.

- Prescrições para ações de comunicação social que necessitem ações neuronais de alta complexidade. Prescrições éticas com base em inteligência artificial.

- Prescrições éticas e de análise crítica sobre as regiões que interagem no processo comunicativo em relação aos efeitos comunicativos ao desligá-las.

As possibilidades investigativas aqui expostas visam exercitar a capacidade criativa dos pesquisadores em comunicação sem ter como objetivo, claro, o esgotamento do assunto que apenas se inicia nos debates acadêmicos.

\section{CONSIDERAÇõES}

Vimos que a intersecção entre neurociência e comunicação é um espaço de possibilidades investigativas e que um olhar mais acurado poderá alavancar as perspectivas em relação ao futuro da comunicação e poderá somar aos esforços já conduzidos em nosso campo.

Consideramos que há possibilidade da criação de linhas de pesquisa, do desenvolvimento de centros de estudos, ou grupo de estudos, que tenham como foco a relação entre neurosciência e comunicação. Consideramos ainda que alguns sublinhas investigativas são possíveis diante dos avanços específicos: neurosciência da mídia; neurosciência da propaganda; neurosciência do jornalismo; neurosciência das relações públicas; neurosciência a comunicação pública; neurociência do entretenimento entre outras.

Cabe ressaltar que o prefixo "neuro" tem se tornado uma espécie de salvoconduto para as intersecções entre neurociência e todos os outros campos do conhecimento. Ao incluir "neuro" na frente de qualquer palavra, dá a impressão, ao leitor leigo de que o assunto é altamente científico embasado em dados neuronais. Portanto, torna-se imprescindível que o investigador que se interesse por esse viés teórico-metodológico use rigorosamente os critérios de prospecção e validação para separar dados e informações pseudocientíficas de resultados válidos.

Muitas indagações surgem das presentes reflexões e podem gerar novos estudos como as considerações éticas do avanço neurocientífico para a comunicação; o papel de governos e outros agentes no empoderamento de descobertas neurocientíficas em comunicação; as consequências sociais entre quem tem acesso e quem não tem acesso aos avanços em neurociência e comunicação; entre tantas outras indagações que nos fazem considerar que verdadeiramente estamos no princípio de uma grande revolução tecnobiossocial.

Diante do exposto, consideramos que a intersecção entre neurosciência e comunicação é um escopo investigativo promissor, carente de estudos rigorosos e metodologias alternativas com amplas possibilidades de contribuições ao avanço científico na busca de soluções criativas para os problemas da vida. 


\section{REFERÊNCIAS}

Bear, M. F.; Connors, B. W. \& Paradiso, M. A. (2002). Neurociências: desvendando o sistema nervoso. Porto Alegre: Artmed. Disponível em: www.jvasconcellos.com.br/fat/FAT_BIO/wpcontent/uploads/2014/09/Livro-de-Fisiologia-Neuroci\%C3\%AAncias-Desvendando-o-siste ma-nervoso-Bear-M.-F.-Connors-B.-W.-Paradiso-M.-A.pdf. Acesso em: 29 nov. 2016.

Castro, C.; Tome, T. \& Barbosa Filho, A. (2005). Midias Digitais. São Paulo: Paulinas.

Cogan, G. B. et al. (2014). Sensory-motor transformations for speech occur bilaterally. Nature, 507(7490): 94-98. Disponível em: www.nature.com/doifinder/10.1038/nature12935. Acesso em: 1 dez. 2016.

Costa, D. C.; Oliveira, J. M. A. P. \& Bressan, R. A. (2001). PET e SPECT em neurologia e psiquiatria: do básico às aplicações clínicas. Revista Brasileira de Psiquiatria, 23: 4-5. Disponível em: www.scielo.br/scielo.php?script=sci_arttext\&pid=S1516-44462001000500003\&lng=pt \&nrm=iso\&tlng=pt. Acesso em: 3 dez. 2016.

Crew, B. (s.d.). Scientists have invented a mind-reading machine that visualises your thoughts ScienceAlert. Disponível em: www.sciencealert.com/scientists-have-invented-a-mind-readin g-machine-that-can-visualise-your-thoughts-kind-of.

Dresch, A. (2015). Design Science Research. Porto Alegre: Bookman.

Duarte, J. \& Barros, A. (2006). Métodos e técnicas de pesquisa em comunicação. São Paulo: Atlas.

Gazzaniga, M.; Ivry, R. B.; Mangun, G. R. (2006). Neurociência cognitiva: a biologia da mente. Porto Alegre: Artemed.

Grau, C. et al. (2014). Conscious Brain-to-Brain Communication in Humans Using Non-Invasive Technologies. PLoS ONE, 9(8): e105225. Disponível em: http://dx.plos.org/10.1371/journal. pone. 0105225 . Acesso em: 4 out. 2016.

Harari, Y. (2015). Sapiens: uma breve história da humanidade. Porto Alegre: L\&PM.

Huth, A. G. et al. (2016). Natural speech reveals the semantic maps that tile human cerebral cortex. Nature, 532(7600): 453-458 . Disponível em: www.nature.com/doifinder/10.1038/nature176 37. Acesso em: 26 nov. 2016.

Kaku, M. (2011). A física do futuro. São Paulo: Rocco.

Kaku, M. (2015). The future of mind: The scientific quest to understand, enhance, and empower the mind. New York: Anchor Books.

Kawano, D. (2014). Publicidade e neurociência: uma revisão bibliométrica. Foz do Iguaçu: Intercom.

Kurzweil, R. (2013). How to create a mind. New York: Penguin.

Lee, H. \& Kuhl, B. A. (2016). Reconstructing Perceived and Retrieved Faces from Activity Patterns in Lateral Parietal Cortex. Journal of Neuroscience, 36(22).

Lemos, A. (2005). Cibercultura e Mobilidade. A Era da Conexão. Rio de Janeiro: Intercom. 
Maclean, P. D. (1990). The Triune Brain in Evolution: Role in Paleocerebral Functions - P.D. MacLean-Google Livros. New York: Plenum. Disponível em: https://books.google.com.br/boo $\mathrm{ks} ? \mathrm{id}=4 \mathrm{PmLFmNdHL} 0 \mathrm{C} \&$ printsec=frontcover\&hl=pt-BR\&source=gbs_ge_summary_r\&ca $\mathrm{d}=0 \# \mathrm{v}=$ onepage $\& \mathrm{q} \& \mathrm{f}=$ false .

McClure, S. M. et al. (2004). Neural Correlates of Behavioral Preference for Culturally Familiar Drinks. Neuron, 44: 379-387.

Nicolelis, M. (2011). Muito além do nosso eu. São Paulo: Companhia da Letras.

Nishimoto, S. et al. (2011). Reconstructing Visual Experiences from Brain Activity Evoked by Natural Movies. Current Biology, 21(19): 1641-1646. Disponível em: http://linkinghub.else vier.com/retrieve/pii/S0960982211009377. Acesso em: 26 nov. 2016.

Reardon, S. (2016). Laser used to control mouse's brain - and speed up milkshake consumption. Nature. Disponível em: www.nature.com/doifinder/10.1038/nature.2016.20995. Acesso em: 18 nov. 2016.

Squirra, S. (org.) (2012). Ciber midias: extensões comunicativas, expansões humanas. Porto Alegre: Buqui.

Squirra, S. C. (2016). A tecnologia e a evolução podem levar a comunicação para a esfera das mentes. São Bernardo do Campo: [s.n.].

Weber, R.; Mathiak, K. \& Sherry, J. (2009). The neurophysiological perspective in mass communication research: theoretical rational,methods, and applications. In M. J. Beatty, J. C. McCroskey \& K. Floyd (orgs.), Biological Dimensions of Communication: Perspectives, Methods, and Research (pp. 41-71). New York: Hampton.

Wolton, D. (2011). Informar não é comunicar. Porto Alegre: Sulina.

Zarkadakis, G. (2015). In our own image. New York: Pegasus. 